\title{
基本有机合成化学和工藝的成就”
}

\section{P. 拉菲柯夫}

基本有机合成或重有机合成是近代化学工業中的主導頜域之一。这一領域们括：合 成那些能矽作为所有其他有机化学工業部門的原料的有机化合物（如合成橡琴、合成杫 脂、人造織維、油港等的原料)，以及合成許多其他工業部鬥所用的杖料，例如溶剂、澗涽

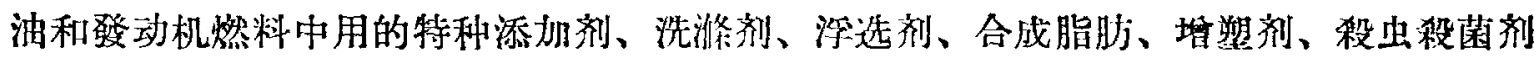

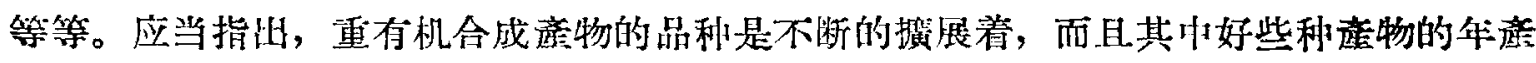

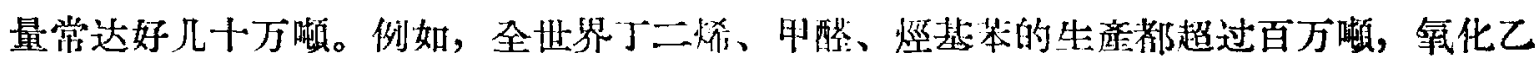

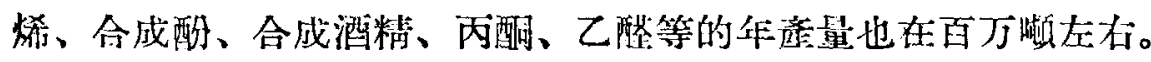

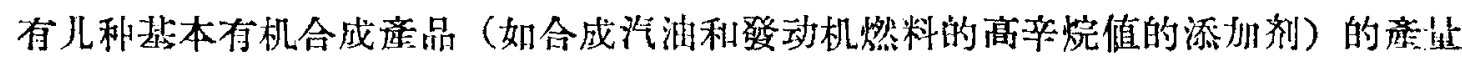
如此之大，以致可以把它們看作与燃料工業相衡接的独立部門。

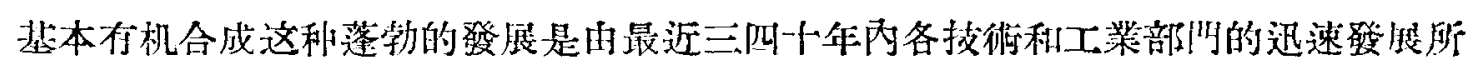

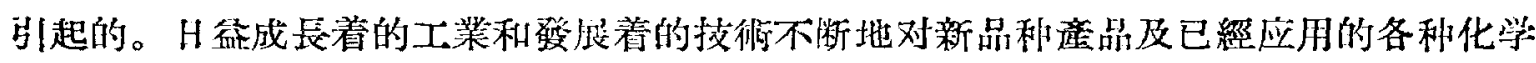

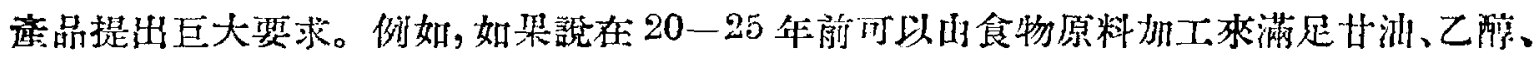

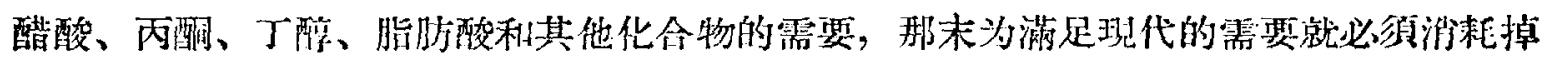

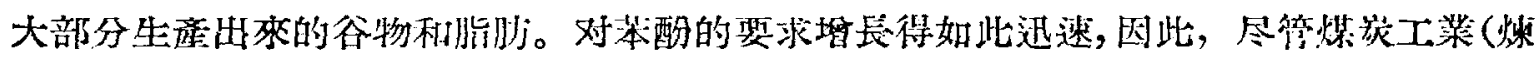

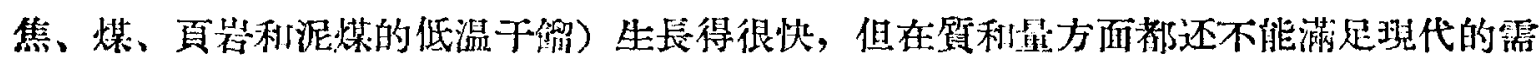

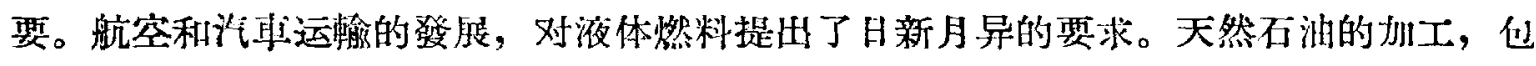
括采用近代的催化裂化方法在內，也不能滿足这些要求，不論是在数辇方面或者是在迹 品种类方面。像这一类的例子我們可以举出儿十个。在所有这类情况下, 萃而有了合成 化学, 它从最价廉和易得的原料出發, 能够制出大是各种各样的、具有需要的性能啲应 品。工業和技術所提出的这些任务的解决, 卖翼上只是一个时問和經济的間題。

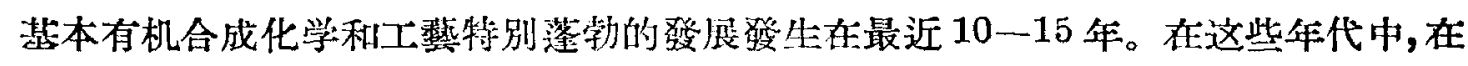

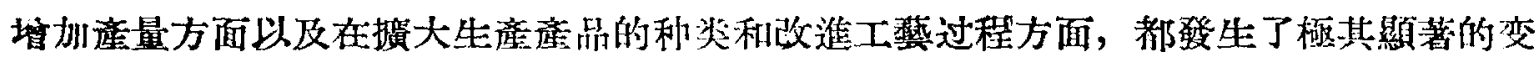
化。

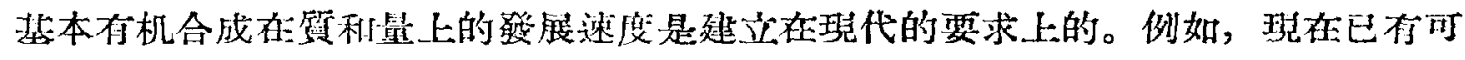

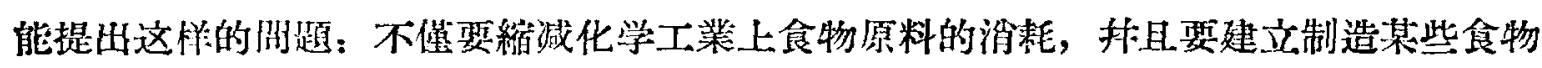
品的工厂（如从石油或煤炭原料制造牊非）。

有机合成化学和工蕉的發展与以下儿个因奖有不可分割的联系: 1. 原料基磷的援大，

* 本交是拉菲柯犬教授于 1955 年 10 月 14 日传天津南开大学和 1955 年 4 月 23 日在沈陽化工棕合研究所

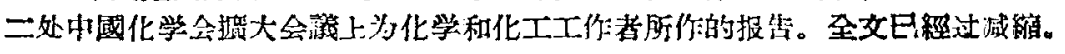

6 月号

科学通振 
2. 新反应和新工筫过程的应用, 3. 布特列洛夫理諭、催化理諭、化学反应动力学与反应 机棬，以及有机化合物反应能力的一般理論概念的舞展。

下面將較詳細地款到有机合成統一的發展过䨂的这三个方面。

\section{有机合成的主要原料}

1. 烟煤、褐煤及面岩加工所得的液体、气体及固体薏物

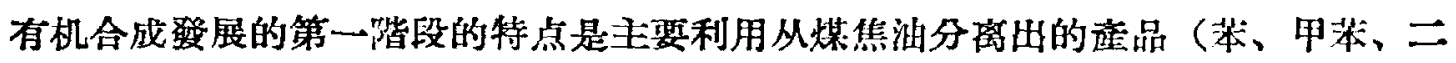

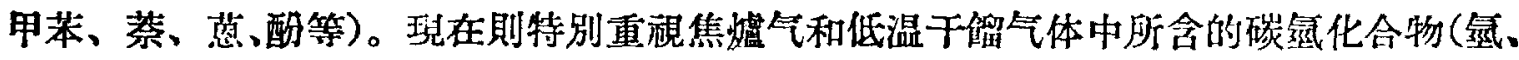
甲烷、乙烷一乙㮁和丙烷一丙烯熘分) 的利用。

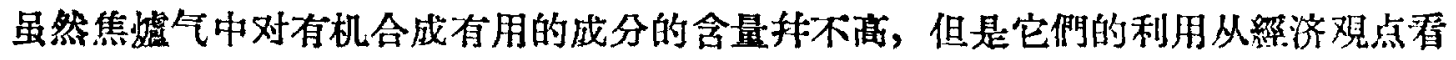
本是完全有道理的。因为, 在近代巨型治金联合企業中, 設有很大的煤焦化学工厂，式 体的总量很可覌。一般焦佣气总量的牛数左右被用作治金燃料, 而另一半縃分济成各利 組分后可用在各种不同的合成上。

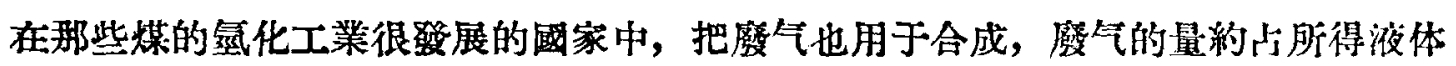
燃料重量的 $10 \%$ 左右。

从煤焦經过电石得到的乙㶧，在基本有机合成原料的比重上少相当重翋的地位。但 是需要指出, 生电石时需要極大的能量, 因面只有在有廉价的电力时这个制取乙炏的

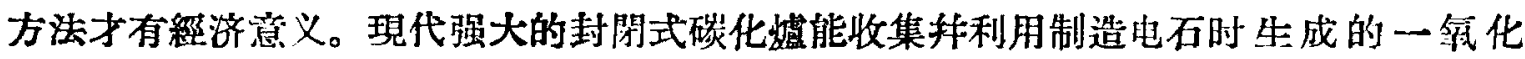
碳, 这就使得生更經济。

近年秝，大大地改進了固体燃料的气化方法。这就使得在有机合成上可以用低筫煤 和生焦來作为制造水煤气的最便宜的來源。在煤气發生器中枝入氯或者水蒸气和鸴的混 合气，能能得到一氯化碳含量高的气体，这种气体適合用于許多合成。

2. 石油加工和人造液体燃料工業的主要道物及副產物

基本有机合成發展初期的特点是利用石油或其熘分热解时生成的不狍和及装秀族碳

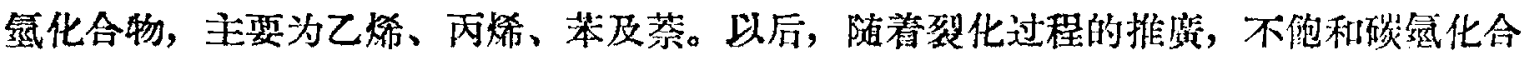
物的利用大大地得到了援展，㪶且还拟定了把各种气体和各石酒馏分（丁烷一丁烯熘分、

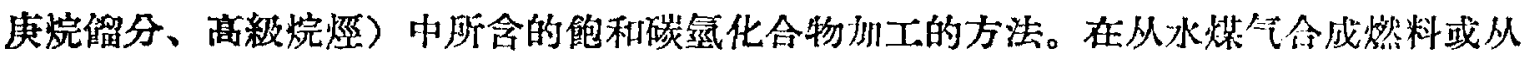
焦油氢化以合成燃料时得到的类似的留分，也找到了席泛的应月。

\section{3. 天然气和石油气}

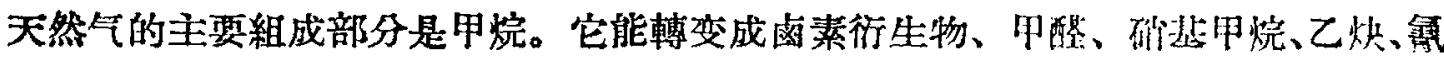
化氢及其他宝貴的化合物。同石油一起放出秝的气体，除含甲烷以外，还有相当数证的 低級飽和碳気化合物（乙烷、两烷、丁烷）。

\section{4. 从農林業屡料和其他植物資源制得的物}

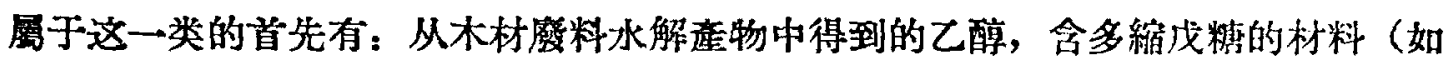

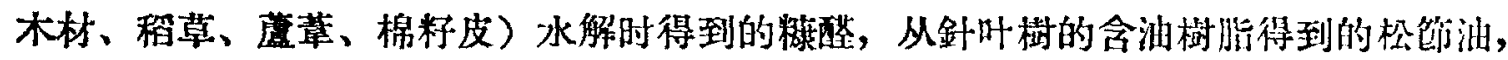

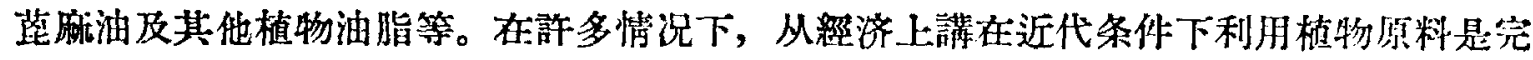

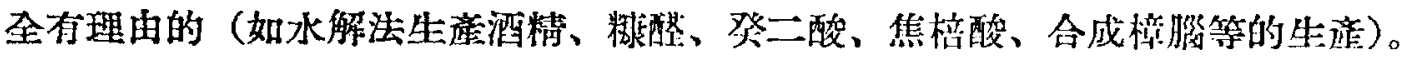

过去这些植物原料利用的極大困难是它們过于分散。現在在很多國家川，些应品和 
木材的加工都相当集中，因此厥料就比較容易得到。

5. 礦物原料、水和空气

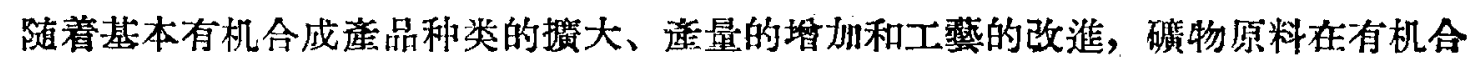
成中的应用大大地㨨大了。那些过去已在有机合成中应用的氯、氧、氯、氮、鉊及碳的 用量地剧䇾地增長了。此外，移、㙳、磷及其他元素的利用，也展开了远大的前途。

没有疑問，在各个不同的國家中，在不同时期內，有机合成的原料閏题要揬照不同

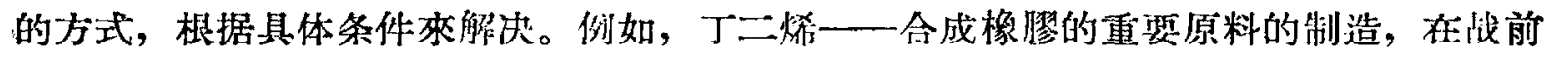

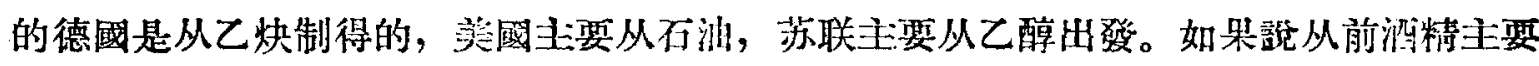
自谷物或馬鈴暮得到，那末現在大部分酒精已經是从乙㶧（經过乙䤊）、烯和其他原料 生产了。在十年前, 甲䤈还只是从甲醇隶制造，而現在極大部分甲䤈的生産是用天然气

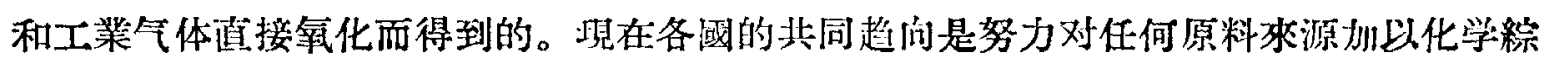
合利用和热能一化学綜合利用, 以及最大限度地利用工農業廢料。

当然, 發展起來的基本有机合成工。業应当有一定的机动性，期随具体条件的改变而

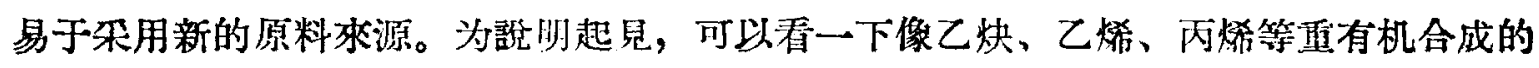
重要特成品利用的主要方向和制造各种击本有机合成產物村可能的代替途径:

\section{乙 炔}

制造隶源：电石、甲烷及其他碳氠化合物气体的热解。

在有机合成中利用的主要方向和可能的代替涂徑;
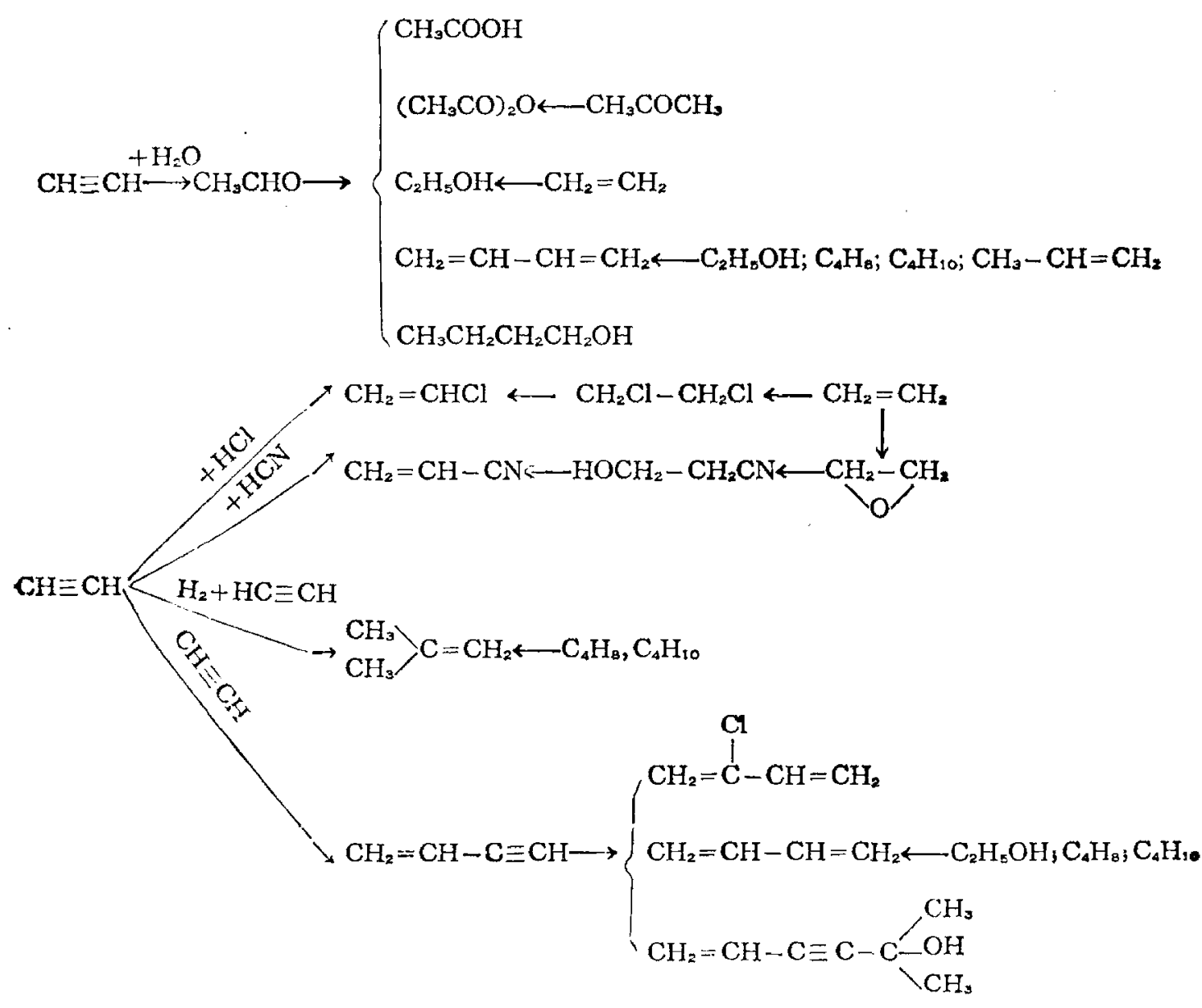

6 月号

科学樋报 


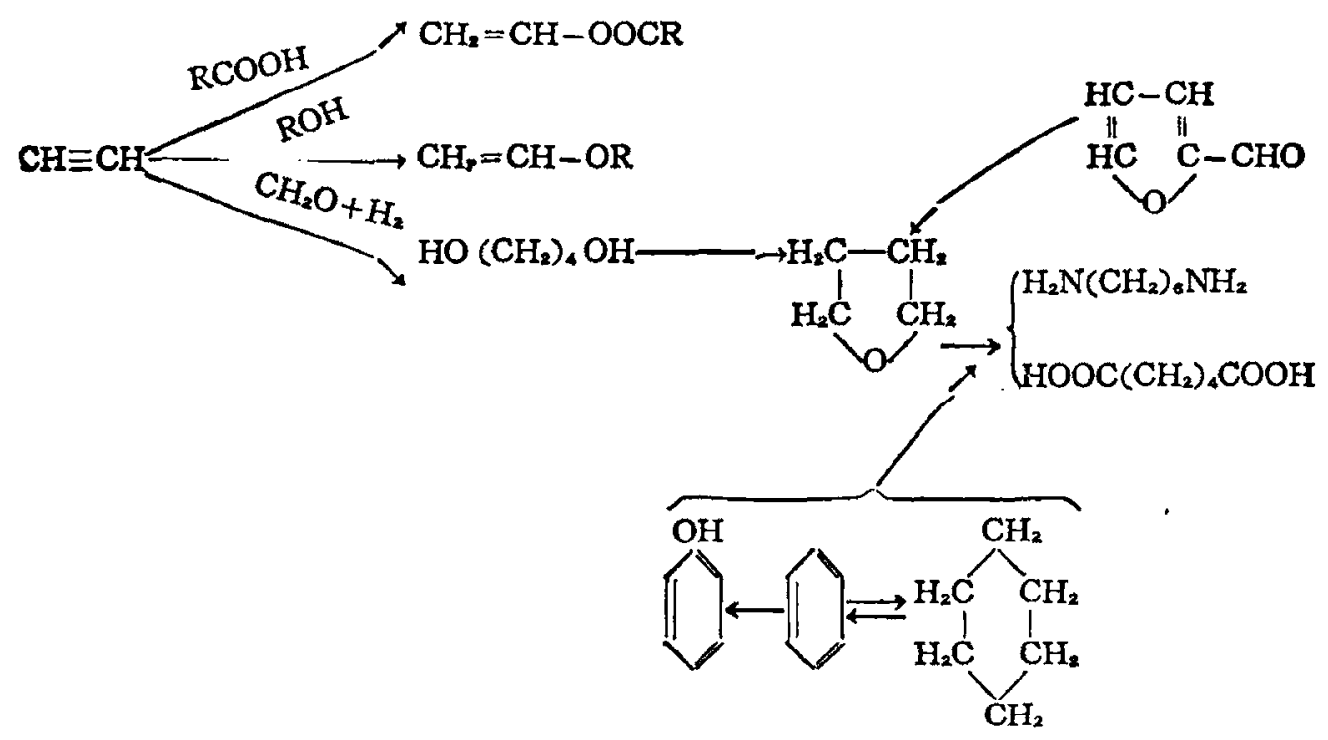

\section{乙 烯}

可能的制造來源：石油加工厂、人造液体燃料生薏和煤的煉焦和低温干熘时所得气 体，石油和焦油热解，甲烷及其他能和碳氢化合物热解，乙㶧氢化，乙醇去水。

利用的方向和可能的代替途徑:

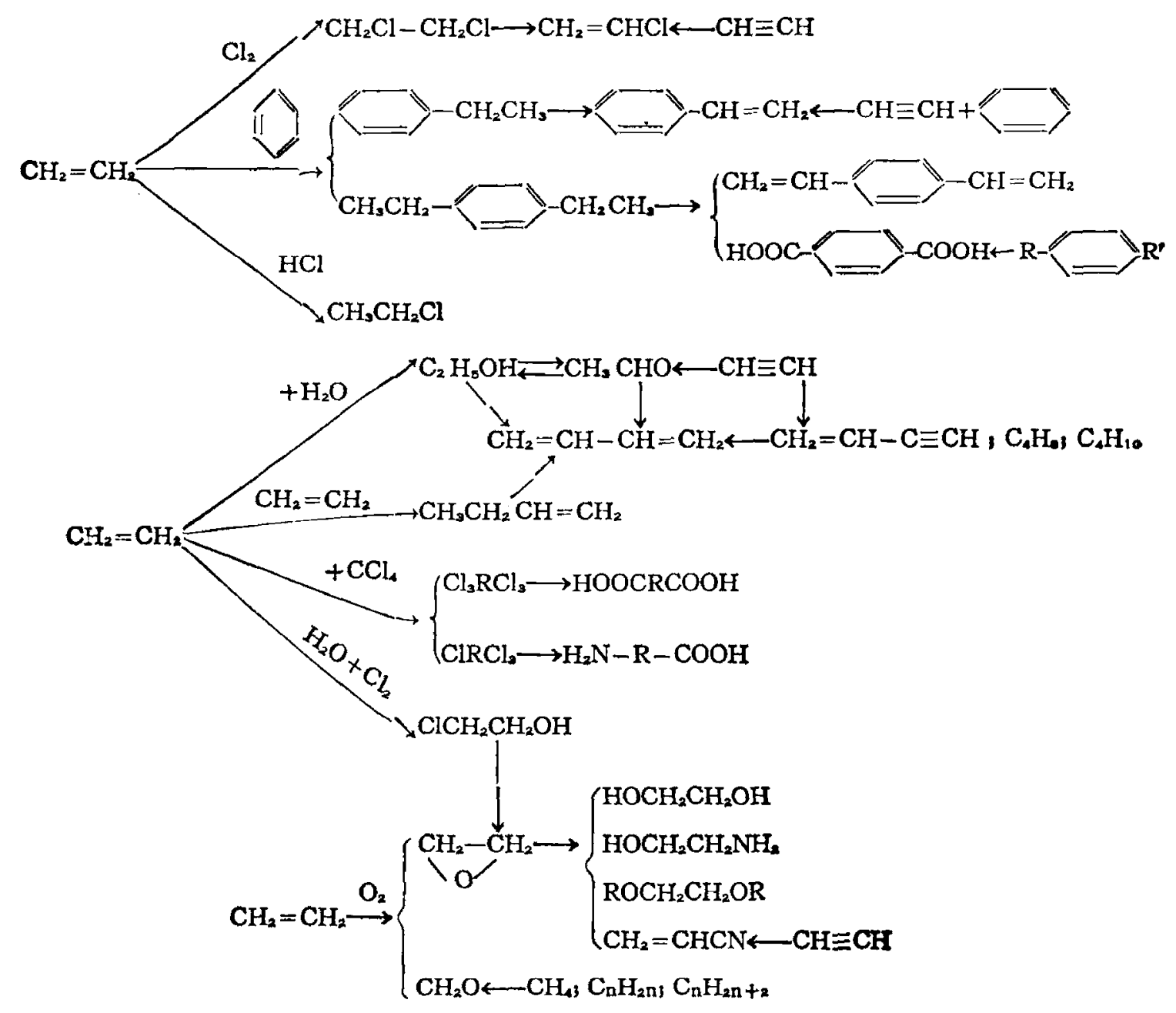




\section{丙 烯}

本源: 石油加工气体副聯物，石油及焦油热解，丙烷热解等。 主要利用途徑及可能的代替途徑:

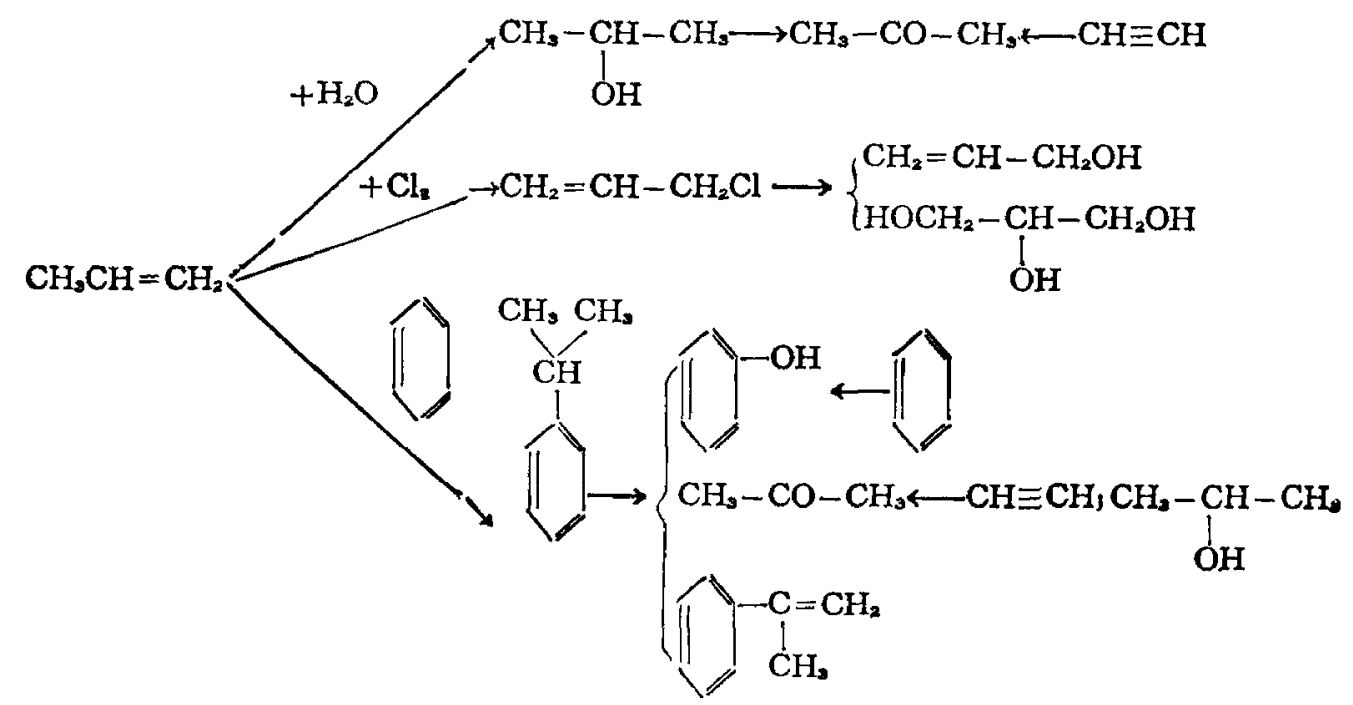

上迅的典型例子指出，根据各个國家和地区的具体条件，解决重有机合成的原料問 題时，可以从网方面考虚。

（1）合理地选摆制取关键性生成品的隶源和方法 (乙烯、乙炔等)。

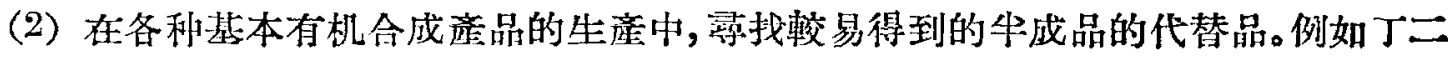

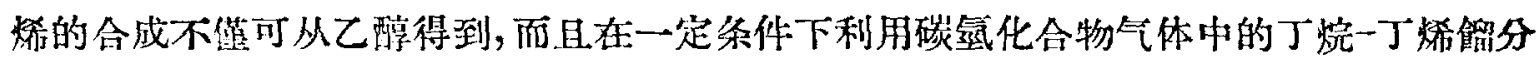
制取更为有利。

应当指出，在有机合成的原料資源有限的地区，把石油加工工厂的气体叠合成汽油 是不合適的。在然料的总量中，叠合汽油占的比重很小。而从这些气体 (特别是 $\mathrm{C}_{2}-$

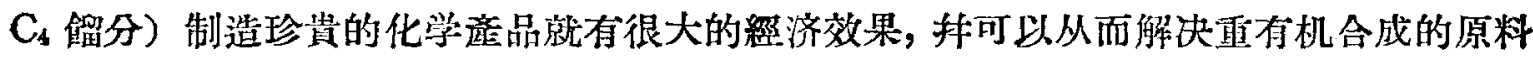
問題。

原料的选擇，在很大程度上还决定于各科过程發展的完善程度，而后者又决定于研 究工作的情况。

新反应的發現和已知反应的深入研究，在訢多情况下，由于有了另外的原料棣源和

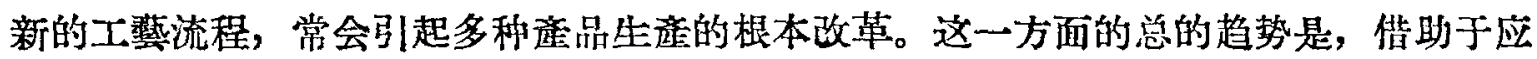
用那些可用催化、光化等活化方法以实現連緽过程的反应，爽强化操作过程及提高其效

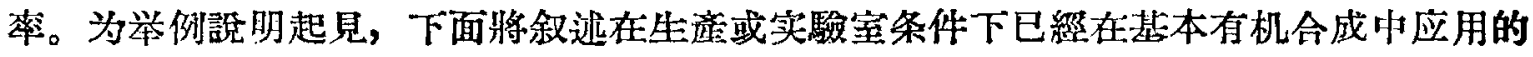
一些反应。

\section{1. 氧化反应}

很久以來，氧化反应就已在有机化学中廣泛应用于有机化合物的合成及其結䟵的确

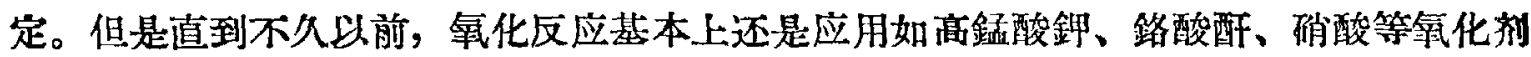
來淮行的。由于氧化剂的价格很高，抹需型耐腐玲的設备，因此一直長期阻碍着氧化反

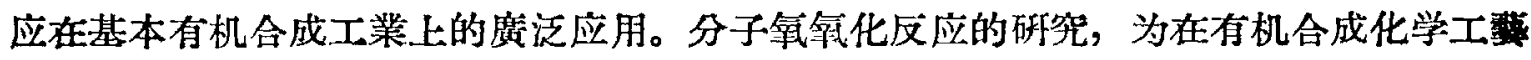
上監泛应用空气或氧气开辟了道路，在很多情况下，使生座这种或那种连品时能改用 
比較更易得的原料和应用更完善的㙅作过程。例如，現在不僅可以从甲䤃氧化桃制得甲

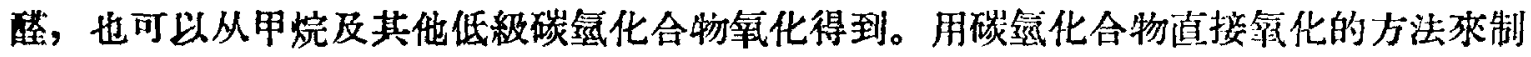

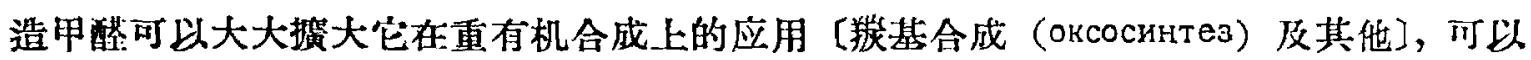

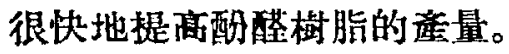

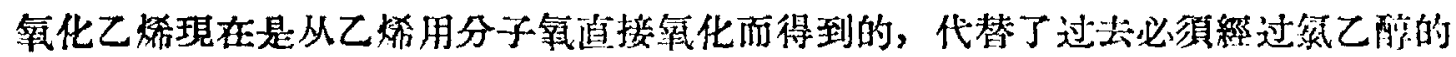

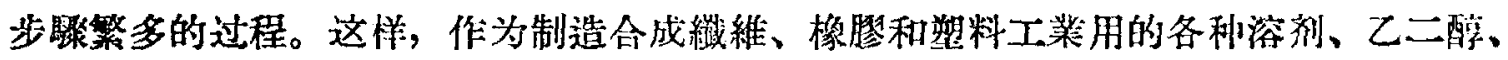
氮基䣼、丙烯腈、聚酷等原料的氧化乙烯就成为極其易得的座品了。

用分子氧在加压下把苯直接催化氧化成苯酚的方法已䌡研究出桃了。

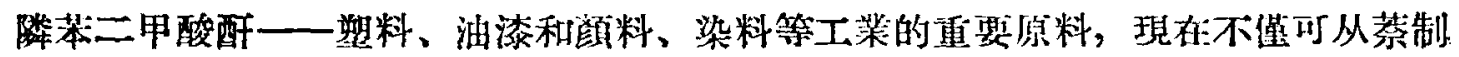
得, 还可以从隣二烴基苯用催化羡化的方法制得。

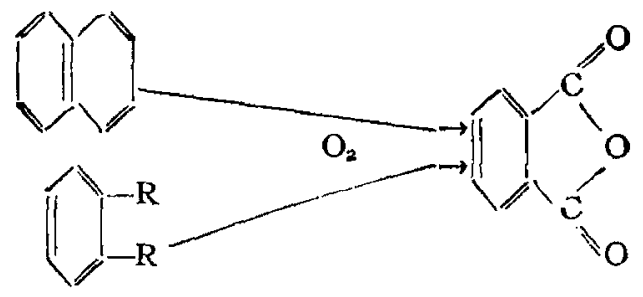

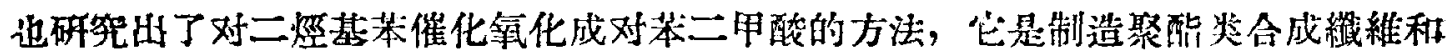
塑料朋的原料。<smiles>[R]OOC(=O)C1=CCC(C(C)=O)C=C1</smiles>

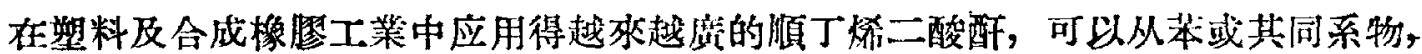

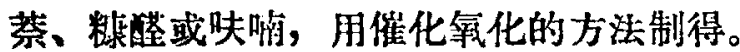

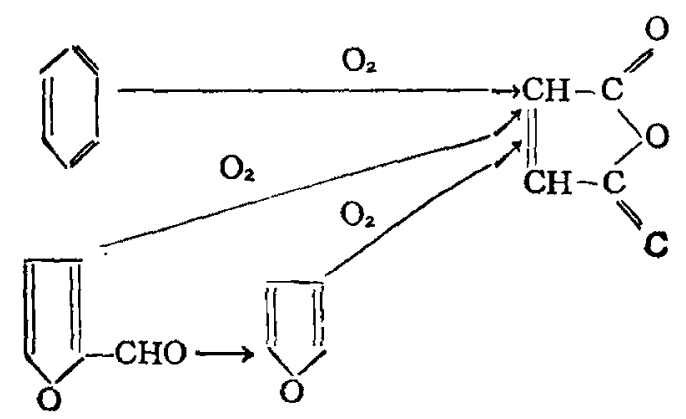

在很多國家中，已絓掌握了由酶級烷烴得到工業用的各种脂助酸混合物以及醇酸或 醇类的催化氧化过程。也已經有了合成食用胎班的工厂。

分子氧的液相氧化反应，展泛用于从异丙苯同时制'得苯酷及丙酮。 


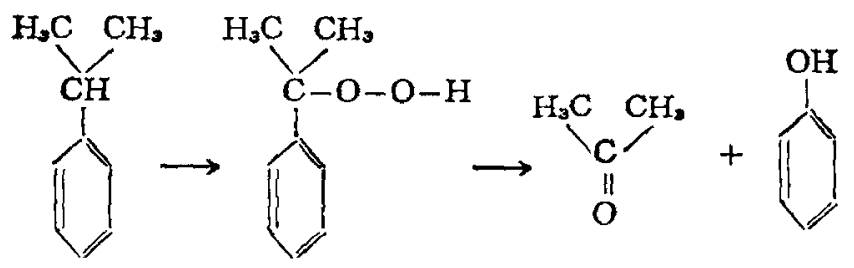

分子氧氧化作用蔍泛地应用于制造各科碳氢化合物的过氯化氢物，这些化合物是生

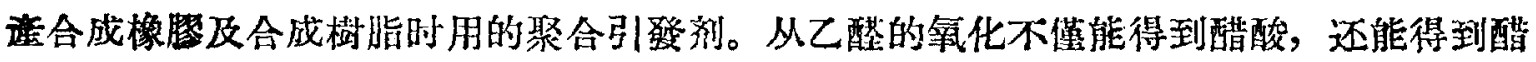
睃酶。

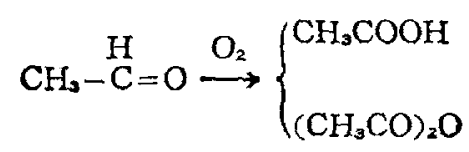

㨁接法制造醋酸䣶的掌握，使醋酸緎維素和烯类聚合物能在塑料、合成緎維、电影 膠片工業中得到更庭泛的应用。

碳氠化合物氯化裂解区应的研究証明，不論反应中有催化剂或沒有催化剂，氧的存 在都可以大大加速去经反应。这样就有可能应用氧化去鿫來合成各科类型的不鵤和化合 物（乙烯、丁烯、 $\alpha$-甲基苯乙烯等)。

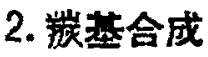

我們知道，早在牛世紀前法伏尔斯基院士就發現了在粉狀䔩性釬存在时丙酮和乙炔 的加成反应:<smiles>C=[C+]C(C)OC=CC(C)(C)O</smiles>

在最近 10-15 年中，許多國家都应用这些反应对各种含张基的化合物和各类有机 化合物進行了詳細的砸究。由于努力研究的結果，掌握了从乙炔、烯类、醇奖和最簡單 的含狶基化合物出發的最重要物的合成方法。可以用下列反应作为例子。

$$
\begin{aligned}
& \mathrm{CH} \equiv \mathrm{CH} \stackrel{\mathrm{CH}_{2} \mathrm{O}}{\longrightarrow} \mathrm{HOCH}_{2}-\mathrm{C} \equiv \mathrm{C}-\mathrm{CH}_{2} \mathrm{OH} \\
& \mathrm{ROH} \stackrel{\mathrm{CO}}{\rightarrow} \mathrm{RCOOH} \\
& \mathrm{CH}_{2}=\mathrm{CH}-\mathrm{C} \equiv \mathrm{CH} \frac{\mathrm{CH}_{3} \mathrm{COCH}_{3}}{\rightarrow} \rightarrow \mathrm{CH}_{2}=\mathrm{CH}-\mathrm{C} \equiv \mathrm{C}-\stackrel{\mathrm{C}-\mathrm{OH}}{{ }_{\mathrm{CH}}} \\
& \mathrm{CH}_{2}=\mathrm{CH}-\mathrm{CH}_{3} \stackrel{\mathrm{CH}_{2} \mathrm{O}}{\longrightarrow} \mathrm{CH}_{2}=\mathrm{CH}-\mathrm{CH}_{2}-\mathrm{CH}_{2} \mathrm{OH} \\
& \left.\right|_{\mathrm{O}} ^{\mathrm{CH}_{2} \mathrm{C}} \stackrel{\mathrm{CO}+\mathrm{H}_{2} \mathrm{O}}{\rightarrow} \mathrm{HOOC}\left(\mathrm{CH}_{2}\right)_{4} \mathrm{COOH}
\end{aligned}
$$

\section{3. 催化血化和去勧}

在溶剂中或气相中，从乙炔的氧化可以制得適合聚合用或其他合成用的高漫度乙烯。 


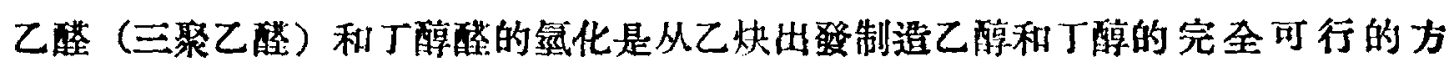
法:

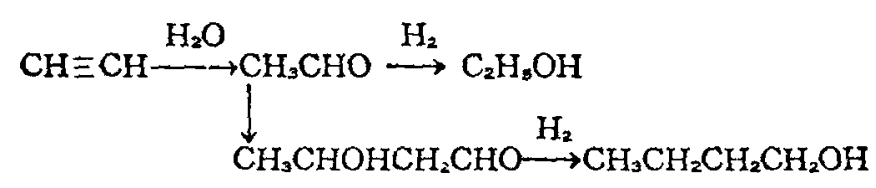

羫酸酯类的聯化度泛应用于制造相应的商級醇类和二元醇;

$$
\begin{aligned}
& \mathrm{RCOOR} \stackrel{\mathrm{H}_{2}}{\longrightarrow} \mathrm{RCH}_{2} \mathrm{OH}+\mathrm{ROH} \\
& \text { ROOCR'COOR } \stackrel{\mathrm{H}_{2}}{\longrightarrow} \mathrm{HOCH}_{2} \mathrm{R}^{\prime} \mathrm{CH}_{2} \mathrm{OH}+2 \mathrm{ROH}
\end{aligned}
$$

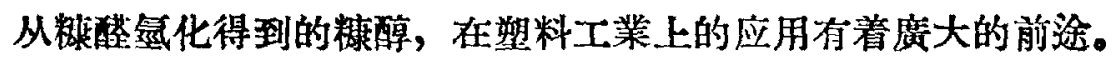<smiles>OCc1ccc(C=Cc2ccco2)o1</smiles>

乙㶧的加钅二聚反应是从廉价的乙炔制造异丁烯的完全可行的方法。

$$
2 \mathrm{CH} \equiv \mathrm{CH} \stackrel{\mathrm{H}_{2}}{\longrightarrow} \underset{\mathrm{CH}_{3}}{\mathrm{CH}_{3}}>\mathrm{C}=\mathrm{CH}_{2}
$$

丁㜔和丁烯的去㖇是最有經济意义的合成丁二烯的方法之一。

偶联或化去氢反应顯然有其展闊的前途。此反应举例如下:

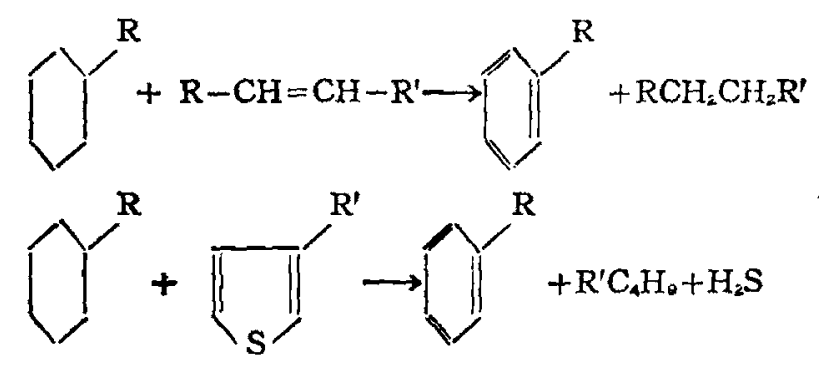

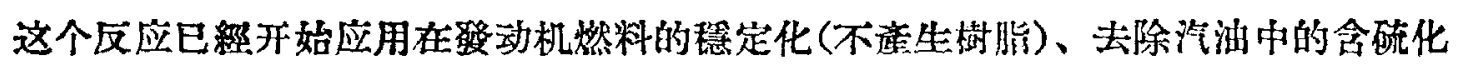

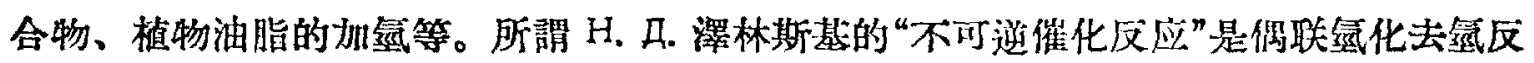

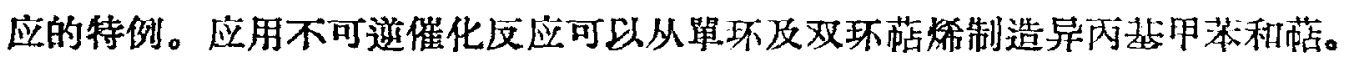

在許多工業过程中，碳氫化合物的去氢是在空气存在下淮行的，这使反应温度可以 大大降低（侧如由甲烷和乙烷制造乙烯或乙炔，自丁烷制造各利丁烯等）。

\section{4. 調節聚合反应}

調節聚合反应正开始在有机合成中席泛应用。侧如, 乙烯在四氯化碳中, 在有过攻 化苯甲酮或其他引發剂存在时的調節聚合反应是按下式進行的。

$$
\begin{aligned}
& \mathrm{R}-\mathrm{R} \longrightarrow 2 \mathrm{R} \cdot \\
& \mathrm{R}^{*}+\mathrm{CCl} \longrightarrow \mathrm{RCl}+\cdot \mathrm{CCl}_{3} \\
& \mathrm{CH}_{2}=\mathrm{CH}_{2}+\dot{\mathrm{C} C l_{3}} \longrightarrow \dot{\mathrm{CH}}_{2}-\mathrm{CH}_{2}-\mathrm{CCl}_{3} \\
& \mathrm{CCl}_{3}-\mathrm{CH}_{2}-\dot{\mathrm{C}_{2}}+\mathrm{CH}_{2}=\mathrm{CH}_{2} \longrightarrow \mathrm{CCl}_{3}-\mathrm{CH}_{2} \mathrm{CH}_{2} \mathrm{CH}_{2} \dot{\mathrm{C}} \mathrm{H}_{2} \\
& \mathrm{CCl}-\mathrm{CH}_{2} \mathrm{CH}_{2} \mathrm{CH}_{2}-\dot{\mathrm{C}} \mathrm{H}_{2}+\mathrm{CCl}_{4} \longrightarrow \mathrm{CCl}_{3}-\left(\mathrm{CH}_{2}\right)_{4}-\mathrm{Cl}+\dot{\mathrm{CCl}},
\end{aligned}
$$




$$
\begin{aligned}
& \text { 2 } \mathrm{CCl}-\mathrm{CH}_{2} \mathrm{CH}_{2} \mathrm{CH}_{2}-\dot{\mathrm{C}} \mathrm{H}_{2} \longrightarrow \mathrm{CCl}_{0}\left(\mathrm{CH}_{2}\right)_{0} \mathrm{CCl}_{3} \\
& \mathrm{CCl}_{3}\left(\mathrm{CH}_{2}\right)_{n-1} \dot{\mathrm{C}_{2}} \mathrm{H}_{2}+\dot{\mathrm{C}}_{3} \longrightarrow \mathrm{CCl}_{3}\left(\mathrm{CH}_{3}\right)_{n} \mathrm{CCl}_{3}
\end{aligned}
$$

改变乙烯和引發剂的浱度能够很好地調節碳鐩的長度。度应后生成的多图化合物的 混合物，很容易分离判轉变成二元酸和氨基酸等。

\section{5. 一些其他例子}

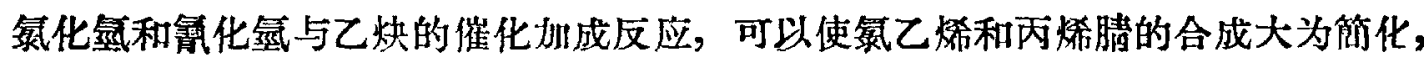
而且可以使这些合成采用新的原料。

$$
\mathrm{CH} \equiv \mathrm{CH} \longrightarrow \mathrm{CH}_{2}=\mathrm{CHCl}
$$

研究出了从甲烷催化命成氮甲烷、二氮甲烷、三氮甲烷和回氮化碳的为法。

从前主要是用于劣香族化合物的确化反应，現在度徒用作碓化低級的烷屬烴。得到 的硝基化合物是很好的溶剂，我可以用作制造醇、醋、酸和其他化合物的原料。

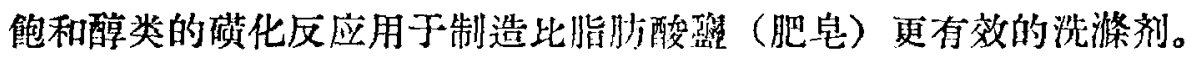

不用湬催化剂的乙炔水化的方法已經得到解沈持应用于工業。研究任了把乙烯直接 催化水化成乙䤃的方法。

应用烴化反应的例子（如莱酚、异烷烴等的烴化）也大大摭大了。

研究出了䳌和与不饱和碳氢化合物的氛衍生物的制造方法, 如 $\mathrm{CCl}_{2} \mathrm{~F}_{2} 、 \mathrm{CClF}=\mathrm{CF}_{2}$ 、 $\mathrm{CF}_{2}=\mathrm{CF}_{2}$ 等。

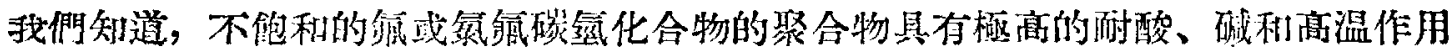
的稳定性。

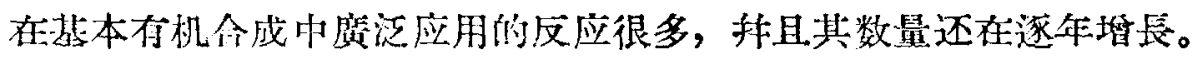

不能不指比应用各利不闰的活化有秃分子的方法的意义。侧如，在制造六绿化体

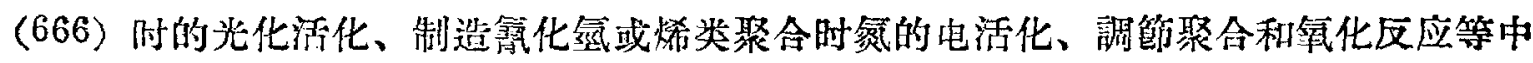
的各种引發剂的应用等。

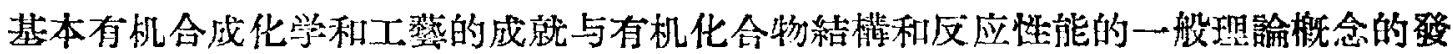

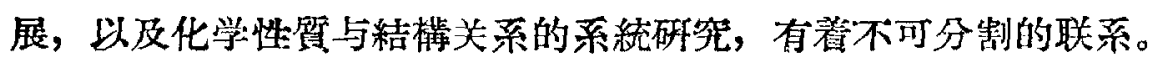

近二十年来, 在謝妙榙夫院士的鐩式反应理踰的研究中, 在唯化理諭的發展和有机化 合物催化轉化的研究中，在化学反应动力学和反应机構的研究中，都有着最㷊出的成就。 需要指出，基本有机合成的發展同时也促淮了理諭有机化学的登展。因为一切理論方面 最重要的成就，是在研究基本有机合成上所用的反应中得到的。

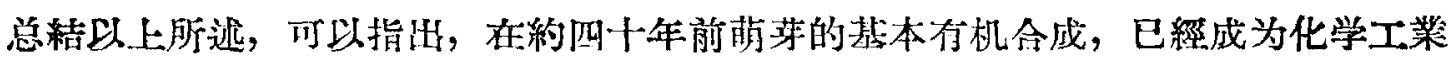

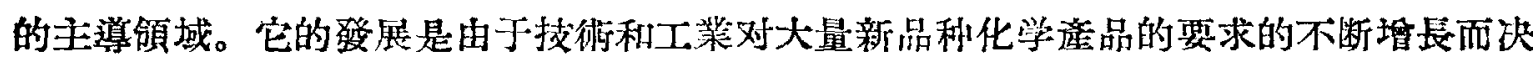
定的。基本有机合成原料的容易得到，以及引等拘發現新的化学反应和寻满已知反应机 構的一般理諭概念的發展，也促進了它的發展。

我們完全有根据認为，将來基本有机合成化学和工藝一定会以更快的涑度發膰，抹 且在短时期內会引起新的質的变化。这个結論的根据，首先就是技術和工業对有机合成

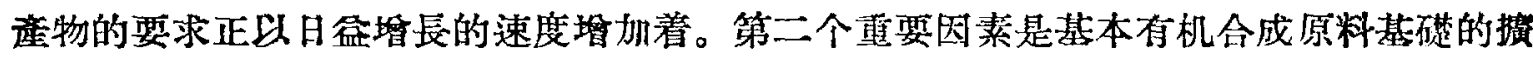
大有着無限的可能性，比其是由于原子能在动力上的应用，水力發电站縌的搌大，以及

6 月号

科学通报 
工䇣过程效率的提即更充分地把原料加以綜合利用)。就在目前,許多國家已有把港 本有机合成工業同石油、煤化学和动力工業結合起隶的明智趋势。

有机化学家和物理化学家的通力合作, 研究催化理諭、鐩式反应理論、結蕃理論及 反应性能的一般問題, 以及化学反应机楢和动力学等等, 对發展基本有机合成论将有 不小的作用。应当指出, 理諭化学的研究是和基本有机合成分不开的, 因为在这一领域

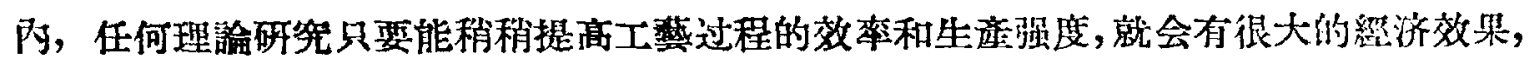
因为这方面品的生座規模是非常大的。应用强放射栈使有机分子活化是發現可应用于 基本有机合成的新型反应的巨大源泉，虽然在这方面的研究还只剛剛开始。应用示路原 子研究化学反应机構和动力学, 也有重大的作用。

解放前的中國，基本有机合成化学和工蓋非常薄弱。因为殖民主义者是不顆意登展中

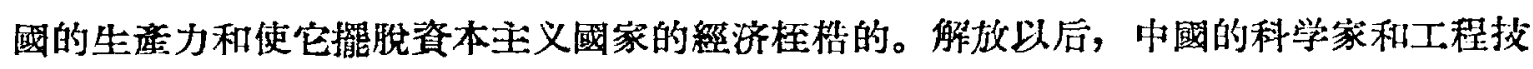
辻人貝皘極地投入了惔复國民經济的工作，在很短的期間內就为發展基本有机合成化学

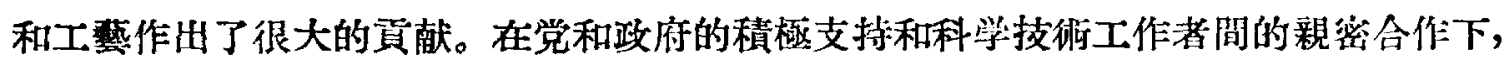

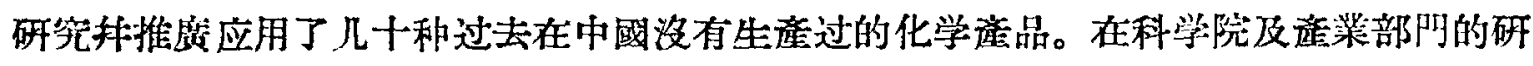

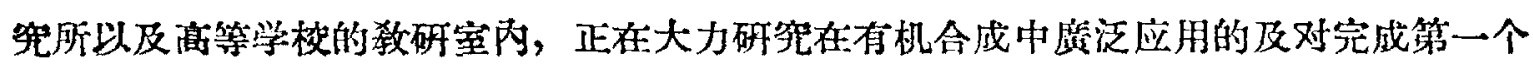
和第二个五年計划有重要意义的許多合成反应。应当指出，不久以前研究出的某些合成 反应, 只是在化学工業很發达的少数國家才完全掌㩧了的, 而且也只不过是最近二三年 的事情。

㳗有疑間，今后还有更艰巨的任务。为了完成發展工業、農業、艾通与屷电、衛生

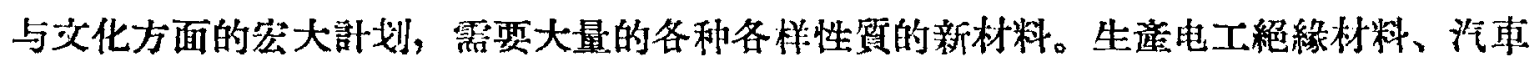
和飛机的染料及各种零件、各秒机器、防治農業害虫的藥剂、合成藥物、染料、湖暴、 部料、有色和黑色金屬的代用品、制造合成油脂、洗滌剂、浮选剂、人造緎維、合成橡 缺和許多其他道物，不可能不發展基本有机合成工業，弡展基本有机合成工業不不能不 淮行相应的科学研究工作*。

为了勝利地完成面前的任务，必須很好地研觉整个国家以及各个地区的紹济發展情 㫛和远景, 以使科学研究工作和試驗工作能符合國家的或金至个別区域的具体要求利可 能。这首先姴求合理地利用國队現有的有机合成的原料。

首先必須实現化学工業和石油工業的充分綜合的發展。为此目的, 又需要研究所有 比较大的石油加工或人造液体燃料工厂的气体的成分。㮛谓研究的結果就可以拟訂合理

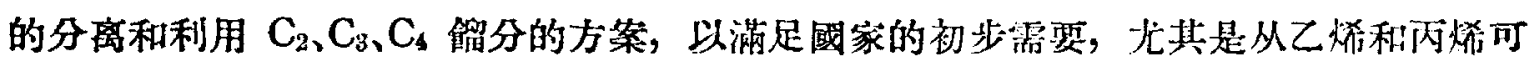
以制得二氮乙烷、氮代乙烯、乙苯、氧化乙秌、乙二醇、异丙醇、氧化丙桥和异丙苯等。 与此同时，最好开展从工厂的丁烷一丁烯嚾分制备丁二烯和异丁烯力面的研究工作。

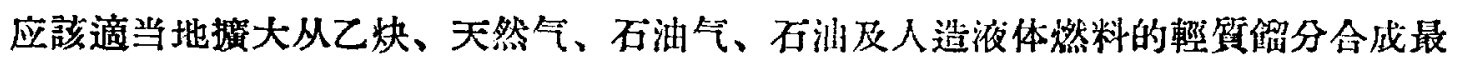

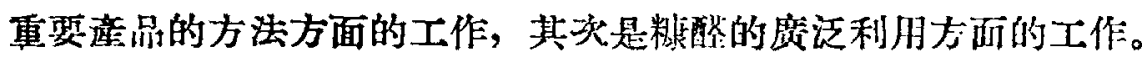

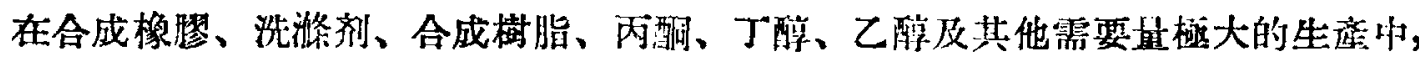

- 作者在北京市及長春市化学会召开的报告会上所作的报告中，提到了今后儿年中基本有机合成化学改工

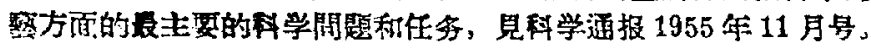


必須努力作到完全不用食物原料。

在國家的不闰的地区，制造同一个道物可以选擇不同的涂俓。为了使各愐工作結果

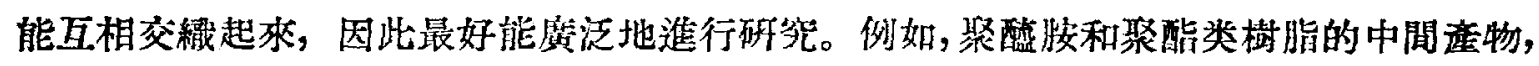

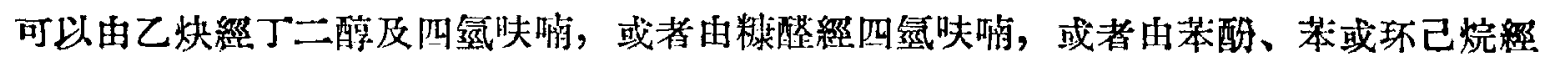
环已酮本制得。最好在不同机路內对所有这些方法都進行研究, 以便在实际建厂时可以 选擇最緅济最合算的方法。选擇生崖乙烯、丁二烯、异丁烯、洗湺剂以及几十秘其他 大量需要的品的生方法时，也应用同样的态度对待。

在研究这种或郘种同拟訂具有重要國民緸济意义的逢品的生潼方法有关的反应时， 沒有深入的理諭研究就不可能得到成就。对發展重有机合成特別重要的是解决下列重要 理諭䦚题: 选擇催化剂的科学基礎的研栄, 有机化合物的催化轉化的研究, 化学反应机 模和动力学的研究，新的分子活化方法的研究。

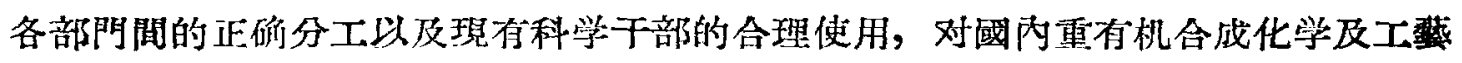
的迅速發展也有着重要的作用。

解放以后的儿年邖, 中國的化学家和工整学家在㓱造性合作和互相帮助方面已經積 累了丰富的經驗。由于科学院和座業部門研究所間的正确分工, 以及在解决某些問題时 吸收商等学校的化学家参加, 因而有些重大的任务在短期內就得到了解决。現在和不久 的将棣，分工的重要原則应該是階段式的研究試駿工作。各部門間的协調会議，能够逐

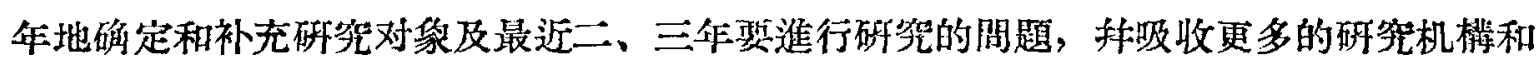
高等学校参加工作。

像基本有机合成化学和工壆这样复雜的科学利技術領域，沒有國际合作，不利用國 外的理論和实踐方面的經驗, 是不问能有成效的發琶的。在利用外國的經驗时, 很主要 的一点是正确地考虑國家在餈源动力和其他因素方面的特点。

不必怀疑，在中國化学家和工䙪学家的密切合作下，在基本有机合成的發展中，像 其他科学和技術領域一样，在不久的将凄就会取得杰出的成就。

〔吕緝青 徐紀平譯〕 